'División de Pediatría, Unidad de Endocrinología Pediátrica, Pontificia Universidad Católica de Chile

${ }^{2}$ Facultad de Medicina, Pontificia Universidad Católica de Chile. ${ }^{3}$ Departamento de Anatomía Patológica.

${ }^{4}$ Departamento de Cirugía Oncológica, Pontificia Universidad Católica de Chile.

aEstudiante de Medicina. Escuela de Medicina. Pontificia Universidad Católica de Chile.

Los autores declaran no tener conflictos de intereses, ni haber recibido aportes financieros de ningún tipo.

Recibido el 13 de septiembre de 2013, aceptado el 17 de marzo de 2014 .

Correspondencia a: Dra. Francisca Grob Lunecke. División de Pediatría. Unidad de Endocrinología Pediátrica. Pontificia Universidad Católica de Chile. Lira $85,5^{\circ}$ piso, Santiago. Código Postal: 8330074 Teléfono (562) 23543630 Fax: 56-2-26384307 fgrob@med.puc.cl

\section{Concordancia de la citología por punción con aguja fina para la detección de cáncer de tiroides en pediatría}

\author{
FRANCISCA GROB ${ }^{1}$, DIEGO CARRILLO ${ }^{2, a}$, \\ ALEJANDRO MARTÍNEZ-AGUAYO ${ }^{1}$, PABLO ZOROQUAIN ${ }^{3}$, \\ ANTONIETA SOLAR ${ }^{3}$, IRINI NICOLAIDES ${ }^{2, a}$, HERNÁN GONZÁLEZ ${ }^{4}$
}

Background: Despite the low frequency of thyroid nodules (TN) in children, one of every four is malignant. Fine-needle aspiration cytology (FNAC) has a high accuracy detecting thyroid cancer. Aim: To evaluate the performance of FNAC in TN in Chilean children to detect thyroid cancer. Patients and Methods: The pathological reports of 77 thyroidectomies and 103 FNAC carried out in patients aged less than 18 years, between 2002 and 2013 were reviewed. In 36 patients aged $15 \pm 2$ years (77\% women), both the reports of the thyroidectomy and FNAC were available. The cytological specimens were reclassified based on Bethesda 2010. The histology was classified as benign (nodular hyperplasia and follicular adenoma, $n=18$ ), or malignant (papillary, follicular and medullar carcinoma, $n=18$ ). The concordance of the cytology with the final biopsy report was calculated. Results: FNAC classified 13 specimens as definitively benign and 13 as definitively malignant. Among these, these concordances with the pathological study of the biopsy was 100\%. Of six cytology tests considered "suspicious for follicular neoplasm" by FNAC, four were benign (67\%), and two malignant (33\%). Of four cytology tests considered "suggestive of carcinoma" by FNAC, one was benign (25\%), and three malignant (75\%). Conclusions: Among the studied children, there was a good concordance between FNAC and surgical biopsies. Therefore a FNAC should be carried out when malignancy is suspected in pediatric patients with a TN.

(Rev Med Chile 2014; 142: 330-335)

Key words: Cytology; Child; Thyroid neoplasms; Thyroidectomy.

4 n la población pediátrica, los nódulos tiroideos (NTP) son una entidad poco frecuente, con una prevalencia estimada entre 0,2 y $1,4 \%$, siendo casi 10 veces más baja que en el adulto $^{1}$. A pesar de su baja ocurrencia, la importancia radica en que uno de cada cuatro NTP son malignos $^{2,3}$, a diferencia de $15 \%$ de malignidad reportado en la población adulta ${ }^{4}$. El cáncer de tiroides representa 1,5 a $3 \%$ de las neoplasias en pediatría. Su incidencia se ha estimado en 0,54 por 100.000, sin embargo, esta parece ir en aumento a una tasa de $1,1 \%$ por año, probablemente relacionado a la exposición a radiación ${ }^{5}$. Antes de la pubertad, la incidencia es igual en ambos sexos, pero luego es más frecuente en mujeres ${ }^{6}$.

La presentación del cáncer de tiroides en la edad pediátrica es más agresiva que en el adulto, se caracteriza por comprometer linfonodos regionales y presentar metástasis pulmonares más frecuentemente ${ }^{7}$. Por tal motivo, un diagnóstico y tratamiento precoz mejoran el pronóstico de esta patología. 
Frente al diagnóstico clínico de un NTP se debe obtener las siguientes hormonas: tiroestimulante (TSH), tetrayodotironina (T4) total y libre (T4 libre) y una ecografía cervical por un radiólogo experimentado. Si la TSH se encuentra suprimida se debe descartar la presencia de un adenoma tóxico con un cintigrama tiroídeo.

Existen ciertas características ecográficas que orientan a malignidad ${ }^{8}$, pero éstas por sí solas no son capaces de diferenciar entre las lesiones benignas y malignas. Por esta razón, frente a la sospecha clínica o ecográfica de malignidad se debe realizar una punción con aguja fina $(\mathrm{PAF})^{9}$ y sus resultados son reportados de acuerdo a las 6 categorías diagnósticas de la clasificación de Bethesda $^{10}$ (Tabla 1), que unifica la terminología para las biopsias tiroideas. Este sistema ha sido validado en pediatría como una prueba sensible para excluir malignidad en esta población ${ }^{11}$.

La PAF es un método de tamizaje importante en los nódulos tiroideos y entrega información sobre riesgo de cáncer para guiar el manejo (Tabla 2). La citología tiroidea por PAF está establecida como parte de la evaluación de rutina de los nódulos tiroideos en adultos y se caracteriza por una alta precisión diagnóstica ${ }^{12,13}$. En pediatría, la utilidad de la PAF también ha sido demostrada ${ }^{2,11}$, pero su uso no se encuentra completamente aceptado. El objetivo principal de este procedimiento es distinguir lesiones benignas de malignas y así evitar cirugías innecesarias. Debido al mayor riesgo de malignidad en los NTP, suele preferirse el tratamiento quirúrgico como primera aproximación a esta patología, considerando de manera limitada el aporte de la PAF, en gran parte debido a la falta de consenso sobre el uso de esta técnica en pediatría. Sin embargo, el tratamiento quirúr-
Tabla 1. El Sistema Bethesda para reportar la citopatología de tiroides: categorías diagnósticas recomendadas

I. No diagnóstico o insatisfactorio

a. Fluido quístico

b. Especimen acelular

c. Otros (sangre, artefactos, etc.)

II. Benigno

a. Consistente con nódulo folicular benigno

b. Consistente con tiroiditis linfocitaria en el contexto clínico adecuado

c. Consistente con tiroiditis granulomatosa

d. Otros

III. Atipia de significado incierto o lesión folicular de significado indeterminado

IV. Neoplasia folicular o sospechoso de neoplasia folicular

a. Especifica si existen células de Hurthle

V. Sospechoso de malignidad

a. Sospechoso de carcinoma papilar

b. Sospechoso de carcinoma medular

c. Sospechoso de carcinoma metastásico

d. Sospechoso de linfoma

e. Otros

VI. Maligno
a. Carcinoma papilar de tiroides
b. Carcinoma pobremente diferenciado
c. Carcinoma medular de tiroides
d. Carcinoma indiferenciado (anaplástico)
e. Carcinoma de células escamosas
f. Carcinoma con características mixtas
g. Carcinoma metastásico
h. Linfoma no-Hodgkin
i. Otros

Tabla 2. Riesgo de malignidad y conducta recomendada según clasificación de Bethesda*

\begin{tabular}{|clcl|}
\hline & Categoría diagnóstica & Riesgo de malignidad (\%) & \multicolumn{1}{c|}{ Manejo } \\
\hline I & No diagnóstico & $1-4$ & Repetir punción \\
II & Benigno & $<1$ & Seguimiento \\
III & Atipia de significado incierto (AUS) & $5-10$ & Repetir punción \\
IV & Sospechoso de neoplasia folicular & $15-30$ & Lobectomía \\
V & Sospechoso de malignidad & $60-75$ & Lobectomía o tiroidectomía total \\
VI & Maligno & $97-99$ & Tiroidectomía total \\
\hline
\end{tabular}

*En adultos. 
gico en esta edad presenta una mayor incidencia de complicaciones que en adultos, especialmente a menor edad del paciente ${ }^{14}$. Las complicaciones incluyen la injuria del nervio laríngeo recurrente ( 0 a $40 \%)$ e hipoparatiroidismo permanente $(0$ a $32 \%)$, las que disminuyen si el procedimiento es realizado por un cirujano de alto volumen, definido como aquel que realiza más de 30 cirugías tiroideas al año ${ }^{14}$.

Para que la citología tiroidea por PAF sea adecuada en la población pediátrica, es necesaria la cooperación del paciente para que la punción sea precisa, por esta razón frecuentemente se realiza bajo sedación. Por otra parte, dado que el material aspirado es habitualmente escaso, se requiere de citopatólogos expertos para su interpretación. Nuestro hospital es un centro de referencia terciario en que se realizan alrededor de $1.500 \mathrm{PAF}$ al año. Aproximadamente $1 \%$ de éstas son realizadas en población pediátrica.

Se evaluó en una muestra local de niños sometidos a citología y tiroidectomía, el valor diagnóstico de la PAF en predecir cáncer de tiroides.

\section{Pacientes y Métodos}

La población estudiada estuvo constituida por todos los pacientes menores de 18 años sometidos a una PAF y tiroidectomía total en la Red de Salud de la Pontificia Universidad Católica de Chile, entre los años 2002 y 2013. Se analizó en forma retrospectiva los registros de los resultados de la citología tiroidea por PAF y de la histopatología obtenidos del departamento de anatomía patológica. El estudio fue aprobado por el comité de ética de la Pontificia Universidad Católica de Chile.

La punción de los nódulos fue realizada por 2 operadores expertos bajo visión ecográfica utilizando gel tibio, un ecógrafo Siemens Acuson 2000 y transductores lineales de $12 \mathrm{MHz}$ y $14 \mathrm{MHz}$. Los niños fueron evaluados en posición supina con el cuello en hiperextensión sin uso de sedación. Se realizó la PAF utilizando una aguja $\mathrm{N}^{\circ} 21$. La muestra fue fijada en formalina y enviada a Anatomía Patológica. Posteriormente, la muestra fue centrifugada y procesada mediante técnica de Agar, de acuerdo a procedimientos estándar ${ }^{15}$. Se obtuvieron de cada caso cortes histológicos seriados de la pieza operatoria (al menos 3 niveles) y todas las muestras citológicas evaluadas fueron re- clasificadas de acuerdo al Sistema Bethesda $2010^{10}$. Las tiroidectomías se procesaron de acuerdo a procedimientos estándar ${ }^{15}$ obteniéndose inclusiones en parafina y preparados histológicos.

Los hallazgos histopatológicos de las piezas operatorias se dividieron en "benigno" y "maligno", considerando como benignos adenoma folicular e hiperplasia folicular coloidea; y malignos carcinoma papilar, carcinoma medular $y$ carcinoma folicular.

En el período estudiado, se realizaron $103 \mathrm{PAF}$ y 77 tiroidectomías totales. Se registró la información de los 36 pacientes que fueron sometidos a ambos procedimientos en nuestra institución. A partir de esta información se construyó una base de datos en Excel y se compararon los informes citológicos con el histopatológico, considerado el "gold"standard para el diagnóstico de cáncer de tiroides. Para cada categoría diagnóstica de la clasificación de Bethesda, se calculó el riesgo de malignidad según la siguiente fórmula: Riesgo de Malignidad = Histología maligna/Categoría citológica. Para las clasificaciones de Bethesda "benigna" y "maligna”, además, se calculó el Valor Predictivo Negativo (VPN) y Valor Predictivo Positivo (VPP), respectivamente, según las fórmulas: $V P N=$ Histología benigna/citología benigna; $V P P=$ Histología maligna/citología maligna .

\section{Resultados}

Se analizaron los resultados de las citologías tiroideas por PAF y tiroidectomías en 36 pacientesque fueron sometidos a ambos procedimientos en nuestro centro. La edad promedio fue 15,2 \pm 2,1 años (Rango de edad; 9 a 18 años), 77\% fueron mujeres y no hubo pacientes prepuberales.

Los resultados de la citología tiroidea por PAF fueron: 13 "benignas", 6 "sospechoso de neoplasia folicular", 4 "sugerente de carcinoma" y 13 "malignas" (Tabla 3). La histopatología demostró 18 lesiones benignas (14 nódulos coloideos y 4 adenomas foliculares) y 18 lesiones malignas (17 carcinomas papilares y 1 carcinoma medular) (Tabla 4).

Al comparar el diagnóstico citológico con el histológico (Tabla 5), de los 13 NTP "benignos" en la PAF, 13 fueron también benignos en la histología; para este grupo se calculó el VPN que fue $100 \%$. De los 6 NTP clasificados como "sospecho- 
Tabla 3. Resultados de la serie según la clasificación Bethesda

\begin{tabular}{|clrc|}
\hline B & Diagnóstico citológico & n & \% \\
II & Benigno & 13 & 36 \\
IV & Sospechoso de neoplasia folicular & 6 & 17 \\
V & Sospechoso de malignidad & 4 & 11 \\
VI & Maligno & 13 & 36 \\
\hline Total & 36 & 100 \\
\hline
\end{tabular}

B: Bethesda.
Tabla 4. Diagnóstico histológico

\begin{tabular}{|llrr|}
\hline \multirow{3}{*}{ Benigno } & Diagnóstico histológico & n & \% \\
\cline { 2 - 4 } Maligno & Hiperplasia coloidea & 14 & 39 \\
& Adenoma folicular & 4 & 11 \\
& Carcinoma papilar & 17 & 47 \\
& Carcinoma folicular & 0 & 0 \\
\cline { 2 - 4 } Total & Carcinoma medular & 1 & 3 \\
\hline
\end{tabular}

Tabla 5. Resultados citológicos e histológicos de los 33 nódulos tiroideos pediátricos estudiados

\begin{tabular}{|c|c|c|c|c|c|c|}
\hline & \multirow{3}{*}{ Citología7 } & \multicolumn{5}{|c|}{ Histología } \\
\hline & & \multicolumn{2}{|c|}{ Benigno } & \multicolumn{2}{|c|}{ Maligno } & \multirow{2}{*}{$\begin{array}{c}\text { Riesgo reportado } \\
\%\end{array}$} \\
\hline & & $\mathbf{n}$ & $\%$ & $\mathbf{n}$ & $\% *$ & \\
\hline$\|$ & Benigno & 13 & 100 & 0 & 0 & $<1$ \\
\hline IV & Sospechoso de neoplasia folicular & 4 & 67 & 2 & 33 & $15-30$ \\
\hline V & Sospechoso de malignidad & 1 & 25 & 3 & 75 & $60-75$ \\
\hline $\mathrm{VI}$ & Maligno & 0 & 0 & 13 & 100 & $97-99$ \\
\hline
\end{tabular}

*Riesgo de malignidad calculado en la muestra.

so de neoplasia folicular", 4 fueron benignos y 2 malignos en la histología. El riesgo de malignidad para esta categoría fue $33 \%$. De las 4 PAF clasificadas como "sugerente de carcinoma", 3 resultaron malignas y 1 benigna. El riesgo de malignidad en la histología para este grupo fue de 75\%. Los 13 NTP informados como "malignos" en la citología por PAF fueron diagnosticados como malignos en la histología; para este grupo, el VPP fue 100\%.

\section{Discusión}

En este estudio determinamos por primera vez en población pediátrica chilena la utilidad de la citología tiroidea por PAF como herramienta diagnóstica preoperatoria en la presentación de nódulos tiroideos. Nuestro hospital es centro de referencia terciario para pacientes del sistema privado de salud en Chile con sospecha de cáncer de tiroides, ya que el cáncer en menores de 15 años está incluido en el grupo de patologías AUGE (Acceso universal de garantías explícitas). En este estudio, no obtuvimos datos de pacientes prepuberales, y la mayoría de éstos fueron de sexo femenino, por lo que la población de estudio probablemente refleje lo que sucede en adolescentes.

El análisis de los datos mostró que la PAF es una prueba muy sensible para la detección de malignidad, con resultados similares a lo reportado previamente en la literatura en adultos ${ }^{10}$. Además, ésta tiene un alto VPN, lo que representa la capacidad de la prueba para predecir con precisión los nódulos benignos. Esto implica, clínicamente, que la PAF es una prueba útil para descartar malignidad en un nódulo tiroideo benigno en niños. Este hecho tiene una gran importancia, ya que permite identificarde mejor manera qué pacientes pueden evitar una tiroidectomía por enfermedad benigna. El uso de la PAF y su rol en la exclusión de malignidad es conocido en la población adulta y se encuentra ampliamente aceptado como el gold standard para el diagnóstico preoperatorio de los nódulos tiroideos ${ }^{16}$.

El 50\% de las tiroidectomías en nuestra serie fueron realizadas frente a la presencia de una citología tiroidea benigna. Lamentablemente, no obtuvimos los datos de la historia clínica y ecográficos de los pacientes para este análisis, pero podemos especular de que en estos casos, 
se decidió el tratamiento quirúrgico debido a la presencia de algún elemento en la historia, ecografía o examen físico sospechoso de malignidad. Los antecedentes familiares de cáncer medular de tiroides, la exposición a radiación, las características del nódulo como tamaño, firmeza y movilidad, deben ser también considerados al momento de decidir la conducta. También es razonable realizar directamente una tiroidectomía en los casos en que la lesión sea muy probablemente maligna, como en aquellos pacientes expuestos a radiación de cuello o antecedentes familiares de cáncer medular de tiroides.

Los pacientes diagnosticados por PAF con cáncer de tiroides presentaron carcinoma papilar en la mayoría de los casos, y uno de ellos resultó ser un cáncer medular de tiroides en la tiroidectomía. Es interesante destacar que este paciente fue correctamente diagnosticado como maligno en la citología. A pesar de que se trata de un solo paciente, la sensibilidad para este caso fue de $100 \%$. La sensibilidad reportada en la literatura para este tipo de neoplasia varía entre $83 \%$ y $94 \%{ }^{17,18}$.

Las lesiones indeterminadas de la citología tiroidea, clasificadas como Bethesda III, IV y V, presentaron un riesgo de cáncer que fue comparable a lo reportado previamente en la literatura. La conducta frente a la mayoría de estos pacientes es la tiroidectomía, pero en la mayoría de los casos se confirma que la enfermedad es benigna. Este hecho tiene especial importancia en la población pediátrica, dado que la cirugía tiene un mayor número de complicaciones, especialmente a menor edad. Además, los pacientes requerirán tratamiento con levotiroxina de por vida. El grupo de pacientes con lesiones indeterminadas en la citología serían candidatos a realizarse el test Afir$\mathrm{ma}^{19}$ con un VPN 93\%, sin embargo, es un test de alto costo, se requiere enviar la muestra a Estados Unidos de Norteamérica y su especificidad es menor a $50 \%$. En un futuro cercano se dispondrá de un test para nódulos indeterminados desarrollado en Chile, que mediante el análisis simultáneo de la expresión de 10 genes, integrados por un algoritmo, muestra un rendimiento prometedor con VPN 98\% y especificidad de 80\% (Comunicación personal del autor, Dr. Hernán González).

Este trabajo proporciona evidencia de que la citología por PAF es un test sensible en población pediátrica y puede ser una herramienta útil para excluir malignidad en pacientes jóvenes. En nues- tro centro, y en este acotado número de pacientes, la correlación de la citología con la histología de la pieza operatoria es comparable a lo reportado en la literatura. La PAF parece ser un procedimiento útil para definir la indicación quirúrgica de un nódulo tiroideo sospechoso de malignidad en pediatría. Por esta razón, sería recomendable el uso de la PAF en este grupo de pacientes cuando las características ecográficas de la lesión y los antecedentes del paciente lo sugieran. Es una técnica segura, con una baja tasa de complicaciones, pero que su éxito depende de la habilidad del operador que toma la muestra y de la experiencia del patólogo que la evalúa ${ }^{20}$.

Agradecimientos: Agradecemos a los Dres. José Miguel Domínguez y Gabriel Cavada, por la revisión crítica de los resultados.

\section{Referencias}

1. Corrias A, Einaudi S, Chiorboli E, Weber G, Crino A, Andreo M, et al. Accuracy of fine needle aspiration biopsy of thyroid nodules in detecting malignancy in childhood: comparison with conventional clinical, laboratory, and imaging approaches. J Clin Endocrinol Metab 2001; 86 (10): 4644-8.

2. Gupta A, Ly S, Castroneves LA, Frates MC, Benson CB, Feldman HA, et al. A standardized assessment of thyroid nodules in children confirms higher cancer prevalence than in adults. J Clin Endocrinol Metab 2013; 98 (8): 3238-45.

3. Niedziela M. Pathogenesis, diagnosis and management of thyroid nodules in children. Endocr Relat Cancer 2006; 13 (2): 427-53.

4. Yassa L, Cibas ES, Benson CB, Frates MC, Doubilet $\mathrm{PM}$, Gawande AA, et al. Long-term assessment of a multidisciplinary approach to thyroid nodule diagnostic evaluation. Cancer 2007; 111 (6): 508-16.

5. Hogan AR, Zhuge Y, Pérez EA, Koniaris LG, Lew JI, Sola JE. Pediatric thyroid carcinoma: incidence and outcomes in 1.753 patients. J Surg Res 2009; 156 (1): 167-72.

6. Rivkees SA, Mazzaferri EL, Verburg FA, Reiners C, Luster M, Breuer CK, et al. The treatment of differentiated thyroid cancer in children: emphasis on surgical approach and radioactive iodine therapy. Endocr Rev 2011; 32 (6): 798-826.

7. Welch Dinauer CA, Tuttle RM, Robie DK, McClellan DR, Svec RL, Adair C, et al. Clinical features associated 
with metastasis and recurrence of differentiated thyroid cancer in children, adolescents and young adults. Clin Endocrinol (Oxf) 1998; 49 (5): 619-28.

8. Domínguez JM, Baudrand R, Cerda J, Campusano C, Fardella C, Arteaga E, et al. An ultrasound model to discriminate the risk of thyroid carcinoma. Acad Radiol 2011; 18 (2): 242-5.

9. Cooper DS, Doherty GM, Haugen BR, Kloos RT, Lee SL, Mandel SJ, et al. Management guidelines for patients with thyroid nodules and differentiated thyroid cancer. Thyroid 2006; 16 (2): 109-42.

10. Cibas ES, Ali SZ. The Bethesda System for Reporting Thyroid Cytopathology. Thyroid 2009; 19 (11): 1159-65.

11. Stevens C, Lee JK, Sadatsafavi M, Blair GK. Pediatric thyroid fine-needle aspiration cytology: a meta-analysis. J Pediatr Surg 2009; 44 (11): 2184-91.

12. Camargo R, Corigliano S, Friguglietti C, Gauna A, Harach R, Munizaga F, et al. Latin American thyroid society recommendations for the management of thyroid nodules. Arq Bras Endocrinol Metabol 2009; 53 (9): 1167-75.

13. Gharib H, Papini E, Paschke R, Duick DS, Valcavi R, Hegedus L, et al. American Association of Clinical Endocrinologists, Associazione Medici Endocrinologi, and European Thyroid Association Medical guidelines for clinical practice for the diagnosis and management of thyroid nodules: executive summary of recommendations. Endocr Pract 2010; 16 (3): 468-75.
14. Sosa JA, Tuggle CT, Wang TS, Thomas DC, Boudourakis L, Rivkees S, et al. Clinical and economic outcomes of thyroid and parathyroid surgery in children. J Clin Endocrinol Metab 2008; 93 (8): 3058-65.

15. Manual de Recomendaciones de Anatomía Patológica para tumores malignos. Ministerio de Salud, Gobierno de Chile. 2010-2011.

16. American Thyroid Association Guidelines Taskforce on Thyroid N, Differentiated Thyroid C, Cooper DS, Doherty GM, Haugen BR, Kloos RT, et al. Revised American Thyroid Association management guidelines for patients with thyroid nodules and differentiated thyroid cancer. Thyroid 2009; 19 (11): 1167-214.

17. Dustin SM, Jo VY, Hanley KZ, Stelow EB. High sensitivity and positive predictive value of fine-needle aspiration for uncommon thyroid malignancies. Diagn Cytopathol 2012; 40 (5): 416-21.

18. Jo VY, Renshaw AA, Krane JF. Relative sensitivity of thyroid fine-needle aspiration by tumor type and size. Diagn Cytopathol 2013; 41 (10): 871-5.

19. Alexander EK, Kennedy GC, Baloch ZW, Cibas ES, Chudova D, Diggans J, et al. Preoperative diagnosis of benign thyroid nodules with indeterminate cytology. $\mathrm{N}$ Engl J Med 2012; 367 (8): 705-15.

20. Kaur J, Srinivasan R, Arora SK, Rajwanshi A, Saikia UN, Dutta $\mathrm{P}$, et al. Fine-needle aspiration in the evaluation of thyroid lesions in children. Diagn Cytopathol 2012; 40 Suppl 1: E33-7. 